\title{
NEW PROPERTIES OF ALL REAL FUNCTIONS*
}

\author{
BY \\ HENRY BLUMBERG
}

\section{INTRODUCTION}

In a former paper $\dagger$ the author communicated a number of properties of every real function $f(x)$; these were stated in terms of the successive saltus functions associated with a given function. The present paper makes no use of the saltus functions, and the new properties are direct qualifications of $f(x)$. Since $f(x)$ is entirely unrestricted,-except, of course, that it is defined $\ddagger$ and therefore finite for every real $x$-these qualifications are consequences of nothing else than that $f(x)$ is a function. A new light is thus thrown upon the nature of a function.

The new properties are of two types, descriptive and metric; the former are concerned with density, and the latter with measure (Lebesgue).

For the sake of greater concreteness of exposition, we shall discuss, for the most part, planar sets and real functions of two real variables.

\section{Descriptive PROPERTIES}

We shall say that a planar set $S$ is an $I$-region (= open set) if every point of $S$ is an inner point of $S$; i.e., no point of $S$ is the limit of a sequence of points not in $S$.

We shall deal with binary relations $\Re$ between $I$-regions and points. $I \Re P$ shall mean that the $I$-region $I$ has the relation $\Re$ to the point $P$. The relation $\Re$ is said to be closed, if the relationships $I \Re P_{n}$ and $\lim _{n \rightarrow \infty} P_{n}=P$ imply $I \Re P$. By a neighborhood of a point $P$, we understand an $I$-region containing $P$; by a partial neighborhood of $P$, an $I$-region of which $P$ is an inner or boundary point. A neighborhood of $P$ is, therefore, also a partial neighborhood of $P$. We have the following

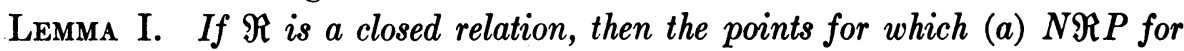
every neighborhood $N$ of $P$, and (b) a partial neighborhood $N_{<}$exists such that $\overline{N_{<} \Re P}$ (i.e., $N_{<} \Re P$ is false) constitute a non-dense (i.e., nowhere dense) set $M$.

* Presented to the Society March 29, 1919, and December 30, 1920.

$\dagger$ Certain general properties of functions, A $\mathrm{n} n$ a l s of $\mathrm{M} \mathrm{at} \mathrm{h}$ e $\mathrm{mat}$ i c s, vol. 18 (1917), p. 147.

$\ddagger$ Even this restriction may be partially dispensed with; cf. Section 2 . 
Proof: Since $\Re$ is closed and $\overline{N_{<} \Re P}$, it follows that no sequence of points $P_{n}$ exists such that $\lim P_{n}=P$ and $N_{<} \Re P_{n}$ for every $n$. Hence a neighborhood $N$ of $P$ exists such that $\overline{N_{<} \Re A}$ for every point $A$ of $N$. In particular $\overline{N_{<} \Re B}$ for every point $B$ of $N N_{<}$, the set of points common to $N$ and $N_{<}$. Since $N_{<}$is a neighborhood of $B$, it follows that no $B$ belongs to $M$. Every neighborhood of a point $P$ of $M$ thus contains a subset, which is an $I$-region, every point of which is outside of $M$.

Now let $z=f(x, y)$ be any given real function of the two real variables $x$ and $y$, defined for every point of the plane $\Pi$, which we take to be the $X Y$ plane of a cartesian system of coördinates. We shall write also $z=f(P)$, where $P=(x, y)$ ranges over $\Pi$. For every pair of real numbers $(x, y)$, there is a single number $f(x, y)$; otherwise $f$ is unrestricted. We define as follows the relation $\Re_{r_{1} r_{2}}$, where $r_{1}$ and $r_{2}$ are two real numbers and $r_{1}<r_{2}$ : If $P$ is a point and $I$ an I-region of $\Pi$, then $I \Re_{r_{1} r_{2}} P$, if and only if an infinite sequence of points $P_{n}$ of $I$ exists, such that

and

$$
\lim P_{n}=P, \quad \lim f\left(P_{n}\right) \text { exists, }
$$

$$
r_{1} \leqq \lim f\left(P_{n}\right) \leqq r_{2} .
$$

It follows that $\Re_{r_{1} r_{2}}$ is closed. For suppose that $\lim P^{(n)}=P$ and $I \Re_{r_{1} r_{2}} P^{(n)}$ for every $n$. Then, for every $n$, there exists a sequence

$$
\left\{P_{m}^{(n)}\right\}, \quad m=1,2, \cdots,
$$

of points of $I$ such that $\lim _{m \rightarrow \infty} P_{m}^{(n)}=P^{(n)}$ and $r_{1} \leqq \lim _{m \rightarrow \infty} f\left(P_{m}^{(n)}\right) \leqq r_{2}$. From the $P_{m}^{(n)}$, we may, in view of the preceding relations, select a sequence of points $\left\{Q_{n}\right\}, n=1,2, \cdots$, such that

$$
\lim Q_{n}=P \quad \text { and } \quad r_{1} \leqq \lim \inf f\left(Q_{n}\right) \leqq \lim \sup f\left(Q_{n}\right) \leqq r_{2} ;
$$

and from the sequence $\left\{Q_{n}\right\}$ a subsequence $\left\{P_{n}\right\}$ such that $\lim f\left(P_{n}\right)$ exists. We then have

$$
\lim P_{n}=P \quad \text { and } \quad r_{1} \leqq \lim f\left(P_{n}\right) \leqq r_{2} .
$$

$\Re_{r_{1} r_{2}}$ is therefore closed.

By the use of Lemma I, we may thus conclude that for every function $f(x, y)$ and every pair of numbers $r_{1}<r_{2}$, the points $P$ of the $X Y$ plane for which (a) $N \Re_{r_{1} r_{2}} P$ for every neighborhood $N$ of $P$ and $(b) \overline{N<\Re_{r_{1} r_{2}} P}$ for some partial neighborhood $N_{<}$of $P$ constitute a non-dense set $T_{r_{1} r_{2}}$. Let $T$ be the sum of all the sets $T_{r_{1} r_{2}}, r_{1}$ and $r_{2}\left(r_{1}<r_{2}\right)$ ranging independently over all the rational numbers. $T$, being the sum of a denumerable number of non-dense sets, is exhaustible* (i.e., of first category according to Baire).

\footnotetext{
* For the terminology of. Denjoy, J o u rnal de Mathématiques, ser. 7, vol. 1 (1915), pp. 122-125.
} 
We shall now use this property of $T$ to obtain a property of all functions. For this purpose, we introduce the notion of dense approach. The function $f(x, y)$ is said to be densely approached at the point $(\xi, \eta)$, or in other words, the point $(\xi, \eta, \zeta), \zeta=f(\xi, \eta)$, of the "surface" $z=f(x, y)$ is said to be densely approached, if for every positive $\epsilon$ there exists a planar neighborhood $N$ of $(\xi, \eta)$, such that the points $(x, y)$ of $N$ for which $\mid f(x, y)$ $-f(\xi, \eta) \mid<\epsilon$ form a dense set in $N$.

We have the following

THEOREM I. For every real function $f(x, y)$ whatsoever, the points of the surface $z=f(x, y)$ that are densely approached form a residual set (= complement of an exhaustible set). Conversely, given any residual set $R$ whatsoever, a function $f(x, y)$ exists that is densely approached at and only at the points of $R$.

Proof.* The points of $\Pi^{\prime}$-i.e., of the surface $z=f(x, y)$-are either isolated or not. The isolated points of $\Pi^{\prime}$ form a denumerable set; therefore the points of $\Pi$ whose corresponding points of $\Pi^{\prime}$ are isolated form a denumerable and therefore an exhaustible set. It is consequently sufficient to prove that the points $P$ of $\Pi$, for which $P^{\prime}$ is a limit point of $\Pi^{\prime}$ but is not densely approached by $\Pi^{\prime}$, form an exhaustible set. Since $P^{\prime}$ is not densely approached, there exists an $\epsilon$ such that for every neighborhood $N$ of $P$, the points $Q$ of $N$ for which $|f(Q)-f(P)|<\epsilon$ are not dense in $N$; i.e., an $I$-region exists in every neighborhood $N$ of $P$ such that $|f(Q)-f(P)| \geqq \epsilon$ for every point $Q$ of the $I$-region. Hence there exists a partial neighborhood $N_{<}$of $P$ such that the inequality $|f(P)-f(Q)| \geqq \epsilon$ holds for every point $Q$ of $N_{<}$. Let now $r_{1}$ be a rational number between $f(P)-\epsilon$ and $f(P)$; and $r_{2}$ a rational number between $f(P)$ and $f(P)+\epsilon$. Then it follows from the inequality $|f(Q)-f(P)| \geqq \epsilon$ for all points $Q$ of $N_{<}$that the set $\left\{Q^{\prime}\right\}_{Q \text { in } N_{<}}$ -i.e., the set of points of $\Pi^{\prime}$ corresponding to the points $Q$ of $N_{<}$-has no point $(\xi, \eta, z)$ as a limit with $r_{1} \leqq z \leqq r_{2}$, where $(\xi, \eta)=P$. Hence $\bar{N} \Re_{r_{1} r_{2}} P$. On the other hand, $P^{\prime}$ is a limit point of $\Pi^{\prime}$ and hence of every $N^{\prime}$ where $N$ is a neighborhood of $P$; hence $N^{\prime}$ has $(\xi, \eta, \zeta)$, with $r_{1}<\zeta$ $=f(\xi, \eta)<r_{2}$, as a limit point, and $P$ is therefore a point of the non-dense set $T_{r_{1} r_{2}}$ associated with the closed relation $\Re_{r_{1} r_{2}}$, and hence of the exhaustible set $T$ which is the sum of all the $T_{r_{1} r_{2}}, r_{1}$ and $r_{2}$ ranging independently over all the rational numbers. The points of $\Pi^{\prime}$ that are not densely approached thus constitute an exhaustible set.

The proof of the converse is immediate. For let $E$ be the exhaustible set complementary to the given residual set $R$. We may write

$$
E=E_{1}+E_{2}+\cdots E_{n}+\cdots
$$

\footnotetext{
* If $S$ is any subset of $I I$, we shall understand by $S^{\prime}$ the set of points of the surface $z=f(x, y)$ corresponding to the points of $\mathcal{S}$. Thus $\Pi^{\prime}$ represents the totality of surface points. Analogously, $P^{\prime}$ will represent the suriace point corresponding to $P$.
} 
where the $E_{n}$ 's are non-dense in $\Pi$ and no pair of $E_{n}$ 's have common points. Let

and

$$
f(P)=\frac{1}{n}, \quad \text { if } \quad P \text { is in } E_{n},
$$

$$
f(P)=2, \quad \text { if } \quad P \text { is in } R .
$$

It is then clear that $f(P)$ is densely approached, if and only if $P$ is in $R$.

Theorem I shows what a remarkable degree of "regularity" every function possesses. This may be better realized, perhaps, by using the following equivalent definition of dense approach. The function $f(x, y)$ is said to be densely approached at $P$, if for every partial neighborhood $N_{<}$of $P, N_{<}^{\prime}$ has $P^{\prime}$ as a limit. We thus get the following equivalent theorem-we omit a restatement of the converse-which shows a kind of "microscopic symmetry" in the structure of the surface $z=f(x, y)$ for unconditioned $f$.

THEOREM I'. With every function $f(x, y)$ whatsoever, there is associated a residual set $R$-dependent on $f$-of the $X Y$ plane such that if $P=(\xi, \eta)$ is a point of $R$, and $N_{<}$a partial neighborhood of $P$, the set $N_{<}^{\prime}$, which consists of the points of the surface $z=f(x, y)$ that correspond to the points of $N_{<}$, has $(\xi, \eta$, $f(\xi, \eta))$ as a limit point.

We pass now to further discriminations in the manner of "approach." The function $f$ is said to be inexhaustibly approached at the point $P$ of $\Pi$, or in other words, $P^{\prime}$ is inexhaustibly approached by $\Pi^{\prime}$, if every neighborhood of $P$ contains, for every $\epsilon>0$, an inexhaustible set of points-i.e., a set that is not exhaustible,- at which $f$ differs from $f(P)$ by less than $\epsilon$.

The function $f$ is said to be exhaustibly approached-we then say also that the point $P^{\prime}=(x, y, f(x, y))$ is exhaustibly approached by $\Pi^{\prime}$-at the point $P=(x, y)$, if it is not inexhaustibly approached at the point; in other words, if a neighborhood $N$ of $(x, y)$ and a number $\epsilon>0$ exist, such that the points of $N$ where $f$ differs from $f(x, y)$ by less than $\epsilon$ form an exhaustible set. If $M$ is any planar set, we shall use, in connection with approach, the expression "via $M$ " to indicate that $(x, y)$ is restricted to range in $M$. Thus " $f$ is inexhaustibly approached at $P$ via $M$ " means that for every neighborhood $N$ of $P$ and every $\epsilon>0$, the set $M N$, which is the aggregate of points common to $M$ and to $N$, contains an inexhaustible set of points at which $f \operatorname{differs}$ from $f(P)$ by less than $\epsilon$. The following definition for exhaustible approach is equivalent to the one above: The point $P^{\prime}$ of $\Pi^{\prime}$ is exhaustibly approached, if a sphere $S$ exists with $P^{\prime}$ as center such that the points of $\Pi$ corresponding to the points of $\Pi^{\prime} S$ form an exhaustible set.

We have the following

Theorem II. For every function $f(x, y)$, there exists in the $X Y$ plane a 
residual set $R$, dependent on $f$, such that if $P$ is a point of $R$, and $N_{<}$a partial neighborhood of $P$, the function $f$ is inexhaustibly, and therefore densely, approached * at $P$ via $R N_{<}$.

Proof. We first note that the points of $\Pi$ where $f$ is exhaustibly approached form an exhaustible set $E_{1}$. For space contains a denumerable dense set

$$
A=\left\{A_{1}, A_{2}, \cdots, A_{m}, \cdots\right\} \text {. }
$$

Associate with each $A_{m}$ a sequence of spheres $S_{m n}, n=1,2, \cdots$, with $A_{m}$ as center and $1 / n$ as radius. Blacken every sphere $S_{m n}$-the interior as well as the boundary-which is such that the totality of points of $\Pi^{\prime}$ contained in it corresponds to an exhaustible subset of $\Pi$. Let $E_{1}$ be the subset of $\Pi$ constituted by the points whicil correspond to points of $\Pi^{\prime}$ that lie in one or more black spheres. Since the number of black spheres is at most denumerable and each black sphere contributes an exhaustible set to $E_{1}$, it follows that $E_{1}$ is exhaustible. If $P$ is a point at which $f$ is exhaustibly approached, $P^{\prime}$ is in at least one black sphere; hence $P$ belongs to $E_{1}$.

Now suppose we remove from $\Pi$ the set $E_{1}$ of points at which $f$ is exhaustibly approached, thus obtaining the set $R_{1}=\Pi-E_{1}$ of points where $f$ is inexhaustibly approached. Since the values of $f$ in an exhaustible set cannot affect the property of inexhaustible approach, every point of $R_{1}$ is inexhaustibly approached via $R_{1}$. Furthermore the points of $R_{1}$, at which $f$ is densely approached via $R_{1}$, iorm a residual set of $\Pi$. For assume for the present that $f$ is bounded,-we shall later drop this restriction. Let $k$ be any number greater than the least upper bound of $f$. Let the function $g(x, y)$ be equal to $k$ at the points of $E_{1}$ and equal to $f(x, y)$ at the points of $R_{1}$. Since the set of points at which $g(x, y)$ is densely approached forms a residual set $R_{g}$ of which only an exhaustible subset can lie in $E_{1}$, there must be a residual set of $\Pi$ in $R_{1}$ at the points of which $g$ is densely approached. This dense approach is clearly valid via $R_{1}$ and for the function $f$. Let $E_{2}$ be the exhaustible subset of points of $R_{1}$ at which $f$ is not densely approached via $R_{1}$. It will now be seen that at every point of the set $R=\Pi-E_{1}-E_{2}, f$ is densely and inexhaustibly approached via $R$, and furthermore that $f$ is inexhaustibly approached at every point $P$ of $R$ via $N_{<} R$, the common part of $N_{<}$and $R$, where $N_{<}$is any partial neighborhood of $P$. Every point of $R$ is inexhaustibly approached via $R_{1}$ and hence via $R$, since an exhaustible set has no effect upon inexhaustible approach. Moreover every point $P$ of $R$ is densely approached via $R_{1}$; i.e., if $N_{<}$is a partial neighborhood of $P$, and $\epsilon$ a positive number, there exists a point $Q$ of $N_{<} R_{1}$ at which $f \operatorname{differs}$ from $f(P)$ by less than $\epsilon$. Since, however, $f$ is inexhaustibly approached at $Q$ via $R$, and since $R$

${ }^{*} f$ is densely approached at $P$ via $M$, which implicitly has $P$ as limit, if for every partial neighborhood $N<$ of $P$ such that $N<M$ has $P$ as limit, the set $\left(N_{<} M\right)^{\prime}$, i.e., the set of surface points corresponding to the points of $N<M$, has $P^{\prime}$ as a limit. 
differs from $R_{1}$ by an exhaustible set, it follows that $Q^{\prime}$ is a limit point of $R^{\prime}$, and therefore $N_{<}$contains a point of $R$ at which $f$ differs from $f(P)$ by less than $\epsilon . \quad P$ is thus densely and inexhaustibly approached via $N_{<} R$.

If $f$ is unbounded, we use the transformation

$$
\bar{f}(x, y)=\frac{f(x, y)}{1+\mid \overline{f(x, y) \mid}} \cdot
$$

The new function $\bar{f}$ is bounded, but the properties of dense and of exhaustible and of inexhaustible approach are preserved by this transformation. We may therefore drop the restriction made above in reference to boundedness.

Theorem III. With every function $f(x, y)$ there is associated (not uniquely, however) a dense set $D$ of the $X Y$ plane such that $f(x, y)$ is continuous, if $(x, y)$ ranges over $D$.

Proof. We shall say that a set of points is " $\epsilon$-spaced" (in the plane $\Pi$ ) if every circle of radius $\epsilon$ contains at least one point of the set. Let $\epsilon_{1}, \epsilon_{2}, \epsilon_{3}, \ldots$ be a decreasing sequence of positive numbers with $\lim \epsilon_{m}=0$. Let $K_{1}$ be any set that is everywhere dense in $\Pi$ and at the points of which $f(x, y)$ is densely approached via $K_{1}$; such a set exists according to Theorem II. Since $K_{1}$ is everywhere dense in $\Pi$, we may select in $K_{1}$ a subset

$$
D_{1}=\left\{P_{11}, P_{12}, \cdots, P_{1 n}, \cdots\right\},
$$

which is isolated (i.e., no point of $D$ is a limit point of $D$ ) and $\epsilon_{1}$-spaced. We enclose each $P_{1 n}$ in a circle $C_{1 n}$ in such a way that (a) no two $C_{1 n}$ 's overlapfor this purpose it is sufficient to make the radius of $C_{1 n}$ less than one half the greatest lower bound of the distances from $P_{1 n}$ to the other points of $D_{1}$; and that (b) $C_{1 n}$ contains a dense subset of $K_{1}$ at every point of which the value of $f$ differs from $f\left(P_{1 n}\right)$ by less than $\epsilon_{1}$ - the existence of such a subset for a sufficiently small radius of $C_{1 n}$ is guaranteed by the fact that $f$ is densely approached at $P_{1 n}$ via $K_{1}$. Denote by $J_{1 n}$ the set of all the points in $K_{1}$ that are in the interior of $C_{1 n}$ and at which $f \operatorname{differs}$ from $f\left(P_{1 n}\right)$ by less than $\epsilon_{1}$, and by $K_{2}$ the subset of $K_{1}$ constituted by all the points of all the $J_{1 n}$ and the points of $K_{1}$ that lie in the interior of $\Pi-\sum_{n=1}^{\infty} C_{1 n}$. At every point $Q$ of $K_{2}$ $f$ is densely approached via $K_{2}$. For if $Q$ is an interior point of $\Pi-\sum C_{1 n}$, this property is obvious. If, however, $Q$ is in $C_{1 n}$, let $\left|f(Q)-f\left(P_{1 n}\right)\right|=\epsilon_{1}-\delta$, $\delta>0 . \quad f$ is densely approached at $Q$ via points of $K_{1}$ at which $f$ differs from $f(Q)$ by less than $\delta$; that is, via points where $f$ differs from $f\left(P_{1 n}\right)$ by less than $\epsilon_{1}$; hence via $K_{2}$. Since $K_{2}$ is everywhere dense and $f$ is densely approached via $K_{2}$ at every one of its points, we may treat it as we $\operatorname{did} K_{1}$. We select an isolated, $\epsilon_{2}$-spaced subset $D_{2}=\left\{P_{21}, P_{22}, \cdots, P_{2 n}, \cdots\right\}$ of $K_{2}$ such that $D_{2}$ contains every point of $D_{1}$. We now enclose each $P_{2 n}$ in a circle $C_{2 n}$ in such a way that (a) $C_{2 n}$ contains a dense subset of points of $K_{2}$ 
where the value of $f$ differs from $f\left(P_{2 n}\right)$ by less than $\epsilon_{2} ;(b)$ no two $C_{2 n}$ overlap; and (c) $C_{2 n}$ lies either entirely within a $C_{1 n}$ or entirely outside of all $C_{1 n}$. Denote by $J_{2 n}$ the set of all the points of $C_{2 n} K_{2}$ at which $f$ differs from $f\left(P_{2 n}\right)$ by less than $\epsilon_{2}$, and by $K_{3}$ the subset of $K_{2}$ constituted by all the points of all the $J_{2 n}$ and the points of $K_{2}$, lying neither in the interior nor on the boundary of any $C_{2 n}$. The set $K_{3}$, like $K_{2}$, is everywhere dense and such that at every point of it $f$ is densely approached via $K_{3}$, and the process may therefore be continued. The set

$$
D_{m}=\left\{P_{m 1}, P_{m 2}, \cdots, P_{m n}, \cdots\right\}
$$

is an $\epsilon_{m}$-spaced, isolated subset of $K_{m}$ and a superset of $D_{m-1}$. The circle $C_{m n}$ enclosing $P_{m n}$ (a) contains a dense subset of points of $K_{m}$ where $f$ differs from $f\left(P_{m n}\right)$ by less than $\epsilon_{m}$, (b) has no points in common with $C_{m p}$ for $p \neq n$ and (c) lies entirely outside of all $C_{p q}, p<m$, if $P_{m n}$ is outside of all $C_{p q}$ for $p<m$, and entirely inside every $C_{p q}, p<m$, containing $P_{m n}$.

We now define the set $D$ of the theorem to be the sum of the $D_{m}$ 's $(m=1$, $2, \cdots)$. That $D$ is dense follows from the fact that $D_{m}$ is $\epsilon_{m}$-spaced. If $P_{m n}$ is a point of $D$, it lies in every circle of a sequence of circles $C_{m n}$, $C_{m+1, n^{(1)}}, \cdots$, such that at the points of $C_{m+p, n^{(p)}}$ that belong to $D$ the function $f$ differs from $f\left(P_{m n}\right)$ by less than $\epsilon_{m+p}$. This proves the asserted continuity.

\section{Generalizations}

So far we have dealt only with real functions of two real variables. It is apparent that the considerations apply equally well to functions of a single variable and to functions of $n$ real variables. The results are, however, essentially of a still more general character. Without entering upon extreme refinements of generalization we may note that the arguments in the preceding section are substantially valid for every function $f(P)$, defined in a set $\mathfrak{\subseteq}$ satisfying the following conditions:

(1) $\subseteq$ is a metric; * that is to say, with every pair of elements $P$ and $Q$ of $\subseteq$ there is associated a non-negative, real number $\overline{P Q}$ (Fréchet's écart) in such a way that if $P, Q$, and $R$ are any three elements of $\subseteq$, then
(a) $\overline{P Q}=\overline{Q P}$;
(b) $\overline{P Q}=0$, when and only when $P=Q$; and
(c) $\overline{P Q}+\overline{Q R} \geqq \overline{P Q}$.

(2) $\subseteq$ is a complete space (vollständiger Raum); $\dagger$ that is to say, if $\left\{P_{1}, P_{2}\right.$,

* Cf., for example, Fréchet, Sur quelques points du calcul fonctionnel, $\mathbf{R}$ e $\mathbf{n} \mathrm{d}$ i c o $\mathbf{n} \mathrm{i}$ del Circolo M a t e matic o di Pal e r o, vol. 22 (1907), p. 1, and Hausdorff, Grundzüge der Mengenlehre, 1914, p. 211.

$\dagger$ Hausdorff, loc. cit., p. 315 . 
$\left.\cdots, P_{n}, \cdots\right\}$ is a "regular" sequence of elements of $\subseteq$, in other words, if for every $\epsilon>0$ there exists an integer $n_{\epsilon}$ such that $\overline{P_{\lambda}} P_{\mu}<\epsilon$ for $\lambda>n_{\epsilon}$ and $\mu>n_{\epsilon}$, there exists a limit element $P$ (i.e., an element $P$ with the property $\lim _{n \rightarrow \infty} \overline{P_{n} P}=0$ ).

(3) $\mathfrak{S}$ contains a denumerable subset that is dense in $\mathfrak{S}$.

(4) $\subseteq$ has no isolated points.

The reader will have no difficulty in introducing in Section 2 the slight modifications that are requisite for making the definitions and the reasoning applicable to a metric, complete space $\subseteq$ with a dense, denumerable subset and without isolated points. By way of illustration, we show* that the distinction between exhaustible and residual sets may always be made for such a space. For suppose $M_{1}, M_{2}, \cdots, M_{n}, \cdots$ are all non-dense subsets of $\subseteq$. Let $\epsilon_{1}, \epsilon_{2}, \epsilon_{3}, \cdots$ be a sequence of positive numbers with $\lim \epsilon_{n}=0$. Since $M_{1}$ is non-dense in $\subseteq$, its complement $\bar{M}_{1}=\Im-M_{1}$ contains a point $A_{1}$ which is in the interior of $\bar{M}_{1}$ (i.e., which is not a limit point of $M_{1}$ ). Let $C_{1}$ be a "sphere" $\dagger$ with center $A_{1}$ and radius $<\epsilon_{1}$ containing only points of $\bar{M}_{1}$. $C_{1}$ contains in turn an interior point $A_{2}$ of $\bar{M}_{2}=\Im-M_{2}$. Let $C_{2}$ be a sphere with center $A_{2}$ and radius $<\epsilon_{2}$ lying entirely in $C_{1}$ and containing only points of $\bar{M}_{2}$. We thus define the sequence of spheres $C_{n}(n=1,2, \cdots)$. The sequence of points $A_{1}, A_{2}, \cdots$ is evidently a regular sequence, since $A_{\lambda}, \lambda>n$, lies in the sphere $C_{n}$, hence its distance from $A_{n}$ is less than $\epsilon_{n}$, and therefore according to property (1) of $\subseteq$ the distance between $A_{\lambda}$ and $A_{\mu}(\lambda>n, \mu>n)$ is less than $2 \epsilon$. Therefore, according to property (2) of $\mathfrak{S}$ there exists a point $A$ of $\subseteq$ which is the limit of $A_{n} . \ddagger A$ lies in every $C_{n}$, hence outside of every $M_{n}$. Therefore $\subseteq$ cannot be represented as the sum of a denumerable set of non-dense sets; that is, $\mathfrak{S}$ is not exhaustible. Since the sum of two exhaustible sets is exhaustible, it follows that a residual set, which is the complement in $\subseteq$ of an exhaustible set, is not itself exhaustible.

Further details of the extension of the ideas of the preceding section to $\mathfrak{S}$ we leave to the reader. We may state the following

Theorem IV. Let $\subseteq$ be any complete, metric space without isolated points and containing a dense, denumerable subset; and $f(P)$ any real function defined for the elements $P$ of $\subseteq . \S$ Then there exists a residual set $R$ such that if $P$ is a point

* Cf., for example, Hausdorff, loc. cit., chap. VIII, Section 9 , especially Theorem VI and p. 328.

$\dagger$ A "sphere" of $\subseteq$ with center $P$ and radius $r$ is the set of points of $\subseteq$ whose écart from $P$ is $\leqq r$.

$\mp$ It is to be observed that if the radius of $C_{n}$ does not approach 0 , no conclusion with reference to the existence of a limit point of $\left\{A_{n}\right\}$ can be made. For this sequence would then not necessarily form a regular sequence; there may therefore be no limit point unless $\mathfrak{S}$ were assumed to be compact. In this case, however, function space would not be an instance for our results, since a "sphere" of function space, while possessing the properties demanded of $\mathfrak{S}$, is not compact.

$\S$ In the terminology of $\mathrm{E}$. H. Moore, $f$ is a function on $\subseteq$ to $A$, where $A$ is the set of real numbers. 
of $R$, and $N_{<}$a partial neighborhood of $P$, the function $f$ is inexhaustibly, and therefore densely, approached at $P$ via $R N_{<}$. Also there exists a dense subset $D$ of $\subseteq$ such that $f(P)$ is continuous if $P$ ranges over $D$.

As particular examples of a complete metric space with a dense denumerable subset and without isolated points, we mention:

(a) Euclidean $n$-space where the écart between two points is the euclidean distance between them.

(b) A perfect subset of a euclidean space.

(c) Hilbert space, that is, the ensemble $\subseteq$ of sequences $\left(x_{1}, x_{2}, \cdots, x_{n}, \cdots\right)$ of real numbers with convergent $\sum_{n=1}^{\infty} x_{n}^{2}$. The écart between two "points" $\left(x_{1}, x_{2}, \cdots, x_{n}, \cdots\right)$ and $\left(y_{1}, y_{2}, \cdots, y_{n}, \cdots\right)$ is defined to be

$$
\sqrt{\left(x_{1}-y_{1}\right)^{2}+\left(x_{2}-y_{2}\right)^{2}+\cdots} \text {. }
$$

The denumerable set consisting of all the sequences $\left(r_{1}, r_{2}, \cdots, r_{n}, 0,0,0\right.$, ...), where the $r$ 's are rational numbers, is dense in $\subseteq$. Furthermore, $\mathbb{S}$ is evidently complete. For if $P^{\prime}, P^{\prime \prime}, \cdots, P^{(n)}, \cdots$ is a regular sequence of points of $\subseteq$, and $P^{(n)}=\left(x_{1}^{(n)}, x_{2}^{(n)}, \cdots\right)$, then for $\epsilon>0$ an integer $i$ exists such that

$$
\sqrt{\sum_{n=1}^{n=\infty}\left(x_{n}^{(p)}-x_{n}^{(q)}\right)^{2}}<\epsilon
$$

for $p>i$ and $q>i$. Hence $x_{n}^{(p)}$ converges to $x_{n}$, say; therefore, $P^{(n)}$ to $\left(x_{1}, x_{2}, \cdots, x_{n}, \cdots\right)$, which, as may be seen, again belongs to Hilbert space. $\mathfrak{\varsigma}$, of course, has no isolated points.

(d) Function space: $\subseteq$ consists of all real continuous functions $f(x)$ defined for $0 \leqq x \leqq 1$. The écart between $f_{1}(x)$ and $f_{2}(x)$ is defined to be $\max \mid f_{1}(x)$ $-f_{2}(x) \mid$; the postulates for écart are then satisfied. That there is a dense, denumerable subset follows from the theorem of Weierstrass that every continuous function is the limit of a uniformly convergent sequence of polynomials, which may be assumed to have rational coefficients and are thus denumerable. Moreover, since the limit of a uniformly convergent sequence of continuous functions is continuous, it follows that a regular sequence has a limit, and therefore $\subseteq$ is complete. $\subseteq$ obviously has no isolated points.

The assumption that $f$ is single-valued may also be dropped without invalidating Theorems I and II of the last section; Theorem III, of course, implies single-valuedness by its very nature. We thus get the following results:

Theorem $\mathrm{I}^{(a)}$. Let $f(x, y)$ be any real function defined for the entire $X Y$ plane and taking at every point at least one value; the number of values may change, however, from point to point and vary from 1 to $c$, the cardinal number of the continuum. Then the points $(x, y)$ such that every surface point $(x, y$, 
$f(x, y))$ is densely approached by the surface $z=f(x, y)$ constitute an exhaustible set.

THEOREM $\mathrm{II}^{(a)}$. If $f(x, y)$ is any real, single- or many-valued function defined for the entire $X Y$ plane, there exists in the $X Y$ plane a residual set $R$, such that if $(x, y)$ is any point of $R$, and $N_{<}$a partial neighborhood of $(x, y)$, then every point $(x, y, f(x, y))$ is inexhaustibly approached via $R N_{<}$.

The proof for these more general results is essentially the same as that for Theorems I and II and is left to the reader.

Theorems $\mathrm{I}^{(a)}$ and $\mathrm{II}^{(a)}$ hold also for any complete, metric space with a dense, denumerable subset and without isolated points.

\section{Metric PROperties}

As in the case of the descriptive properties of Section 1, we shall confine the discussion in this section to planar sets and to one-valued functions of two variables.

Let $S$ be any planar set; $P$, a point of $S ; C_{r}$, a circle (interior and boundary) with $P$ as center and $r$ as radius; $m\left(C_{r}\right)$, the area of $C_{r}$, and $m_{e}\left(S C_{r}\right)$, the exterior Lebesgue measure of the portion of $S$ in $C_{r}$. Then if

$$
\lim _{r \rightarrow 0} \frac{m_{e}\left(S C_{r}\right)}{m\left(C_{r}\right)}
$$

exists and is equal to $l$, we shall say that the exterior metric density of $S$ at the point $P$ is $l$. We have the following

Theorem V.* Let $S$ be any planar set. Then the points of $S$ at which the exterior metric density of $S$ is $\neq 1$, i.e., the points where the exterior metric density either does not exist or does exist and is $<1$, constitute a set of zero measure (Lebesgue).

Proof. If $P$ is a point at which the exterior metric density of $S$ does not exist or exists and $\neq 1$, the

$$
\liminf _{r \rightarrow 0} \frac{m_{e}\left(S C_{r}\right)}{m\left(C_{r}\right)}
$$

must be $<1$. Let $T_{k}$ be the totality of points of $S$ where

$$
\liminf _{r \rightarrow 0} \frac{m_{e}\left(S C_{r}\right)}{m\left(C_{r}\right)}<k<1
$$

The set whose measure we are to prove equal to zero is the sum of $T_{1 / 2}, T_{3 / 4}$, $\cdots, T_{(n-1) / n}, \cdots$; and since the sum of $\boldsymbol{\aleph}_{0}$ sets each of zero measure is again

\footnotetext{
* For the case where only measurable sets are admitted, cf. Lebesgue, Leçons sur l'Intégration, 1904, pp. 124-125; Denjoy, J ournal de Mathématiques, ser. 7, vol. 1 (1915), p. 132; Lusin and Sierpinski, Rendiconti del Circolo Matematico di P a l e r m o, vol. 42 (1917), p. 167; de la Vallée Poussin, Cours d'Analyse, vol. 2, 1912, p. 114. For the linear case of general (not necessarily measurable) sets, cf. Blumberg, B u 11 e $t$ i n of the American Mathematical Society, vol. 25 (1919), p. 350.
} 
of zero measure, it is sufficient to prove that $T_{k}$ is of zero measure. Let $m$ be the exterior measure of $T_{k}$ so that $T_{k}$ may be enclosed, in such a way that every point of $T_{k}$ is an interior point of at least one $C_{n}$, in a sequence of circles $C_{n}, n=1,2, \cdots$, with the sum of their areas $<m+\epsilon$, where $\epsilon$ is arbitrarily small. Associate with every point $P$ of $T_{k}$ a circle $C_{P}$ lying in the interior of one of the circles $C_{n}$, and such that

and, a fortiori,

$$
\frac{m_{e}\left(C_{P} S\right)}{m\left(C_{P}\right)}<k
$$

where

$$
\frac{m_{e}\left(C_{P} T_{k}\right)}{m\left(C_{P}\right)}<k
$$

$$
m\left(C_{P}\right)=\text { area of } C_{P} ;
$$

this is possible because of the assumed property of the points of $T_{k}$. From the circles $C_{P}$ a denumerable number may be extracted having the same totality of interior points, and from this denumerable number, a finite number $C^{\prime}, C^{\prime \prime}, \cdots, C^{(h)}$, that cover $T_{k}$ except for a set of exterior measure $<\epsilon$. We now select from the $C^{(n)}$ a subset of non-overlapping circles as follows. Let $D^{\prime}$ be a $C^{(n)}$ having the maximum area attained by the $C^{(n)}$; remove from the set $\left\{C^{(n)}\right\}$ the circles which intersect $D^{\prime}$, and let $D^{\prime \prime}$ be one of the circles left, and having the maximum area for these circles; remove now all circles intersecting $D^{\prime \prime}$, and let $D^{\prime \prime \prime}$ be one of the circles now remaining and having the maximum area for all circles intersected neither by $D^{\prime}$ nor by $D^{\prime \prime}$. In this way we obtain a set of non-overlapping circles $D^{(n)}$. Let $a_{1}$ represent the area of the portion of the plane covered by one or more $C^{(n)}$, and $a_{2}$ the portion of the plane covered by the $D^{(n)}$; then it follows from the manner in which the $D^{(n)}$ were chosen that

$$
a_{2}>\frac{1}{9} a_{1}
$$

Since every $D^{(n)}$ is a $C^{(n)}$ we have

$$
\frac{m_{e}\left(D^{(n)} T_{k}\right)}{m\left(D^{(n)}\right)}<k
$$

and since the $D^{(n)}$ do not overlap, we may cover the subset of $T_{k}$ that lies in the $D^{(n)}$ by means of circles having a total area $<k a_{2}$. We may thus cover $T_{k}$ by means of circles having as sum of their areas a number less than $k a_{2}+\left(a_{1}-a_{2}\right)+\epsilon$, the first term representing a sufficient amount for the portion of $T_{k}$ in the $D^{(n)}$, the second term, for the portion of $T_{k}$ in the $C^{(n)}$ and outside of the $D^{(n)}$, and the third term, for the portion of $T_{k}$ outside of the $C^{(n)}$. Therefore,

$$
m<k a_{2}+\left(a_{1}-a_{2}\right)+\epsilon=a_{1}-(1-k) a_{2}+\epsilon ;
$$


and in virtue of the inequalities

we have

$$
m-\epsilon<a_{1}<m+\epsilon, \quad a_{2}>\frac{1}{9} a_{1},
$$

$$
m<m+\epsilon-\frac{1}{9}(1-k)(m-\epsilon)+\epsilon,
$$

whence

$$
m<\frac{(19-k)}{1-k} \epsilon,
$$

and therefore $m=0$.

Definition. $\quad N_{<}$is said to be a non-vanishing partial neighborhood of $P$, if the exterior metric density of $N_{<}$at $P$ is $\neq 0$.

We have the following lemma which corresponds to Lemma $I$ in the case of the descriptive properties.

Lemma II. Let $\Re$ be a closed relation as in Lemma I. The points $P$ for

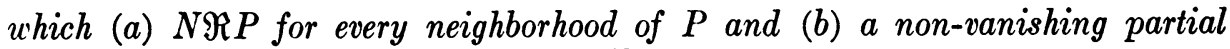
neighborhood $N_{<}$exists such that $\overline{N_{<} \Re P}$ (i.e., $N_{<} \Re P$ is false) constitute a set of zero measure.

Proof. Let $T$ be the totality of points $P$ having the properties $(a)$ and $(b)$. We may then show as in the proof of Lemma $I$ that $\overline{N_{<} \Re B}$ for every point $B$ of $N N_{<}$, which, if $N_{<}$is a non-vanishing neighborhood of $P$, is also a nonvanishing neighborhood of $P$. Every point $P$ of $T$ thus has a non-vanishing neighborhood every point of which does not belong to $T$; therefore at no point of $T$ is the exterior metric density of $T$ equal to $1 ; T$ is therefore of zero measure.

Let $z=f(x, y)$ be a given surface; $P=(x, y)$, a point of the $X Y$ plane; and the relation $\Re_{r_{1} r_{2}}, r_{1}<r_{2}$ (as in the case of Section 1 ), such that $I \Re_{r_{1} r_{2}} P$ when and only when there is a point $(x, y, \zeta), r_{1} \leqq \zeta \leqq r_{2}$, which is a limit of $I^{\prime}$. It follows as before that $\Re_{r_{1} r_{2}}$ is closed. Therefore, by means of Lemma II, we may conclude that the points $P$ for which $(a) N \Re_{r_{1} r_{2}} P$ for every neighborhood $N$ of $P$, and $(b) \overline{N \Re_{r_{1} r_{2}} P}$ for some non-vanishing partial neighborhood of $P$ constitute a set $T_{r_{1} r_{2}}$ of zero measure. Let $T$ be the sum of all the sets $T_{r_{1} r_{2}}$, as $r_{1}$ and $r_{2}, r_{1}<r_{2}$, vary over all the rational numbers; $T$, being the sum of $\boldsymbol{N}_{0}$ sets of measure zero, is itself of measure zero. Now a point of the surface $z=f(x, y)$ is either isolated (and then $(x, y)$ belongs to a denumerable set of points and hence to a certain set of measure zero) or else it is a limit point of the surface, and then $N \Re_{r_{1} r_{2}} P$ for every neighborhood $N$ of $P$, if $r_{1}<z<r_{2}$. We therefore have the following

Theorem VI. Let $f(x, y)$ be any real, one-valued function defined in the entire plane. Then there exists in the $X Y$ plane a set $Z$, dependent on $f$, of measure zero, such that if $(a)(x, y)$ is any point of the $X Y$ plane not belonging 
to $Z$; (b) $N_{<}$, any non-vanishing partial neighborhood of $(x, y)$; and $(c) S$, any sphere with $(x, y, f(x, y))$ as center; then there is at least one point of the surface $z=f(x, y)$ lying in the sphere $S$ and having as projection upon the $X Y$ plane a point in $N_{<}$.

That the theorem becomes false if we omit the restriction that the partial neighborhood $N_{<}$shall be non-vanishing, is seen from the following example. Let $A$ be a planar non-dense perfect set of positive measure, and $B$ its complement. Let $f(x, y)=0$ in $A$ and 1 in $B$. Since $A$ is non-dense and closed, $B$ is an everywhere-dense $I$-region and therefore every point $(x, y)$ of $A$ has as one of its partial neighborhoods a set $N_{<}$, consisting exclusively of points of $B$. For such an $f(x, y),(x, y)$ and $N_{<}$, however, the assertion of the theorem is false, although, since $A$ is not of zero measure, there are points of $A$ not belonging to the alleged set $Z$ of the theorem. Of course, in virtue of Theorem $\mathrm{V}$, the points of $A$, with the exception of those belonging to a set of measure zero, have none but vanishing partial neighborhoods that consist entirely of points of $B$.

It is obvious, as, indeed, the last example shows, that the points at which a function is not densely approached need not be of zero measure. If in passing, however, we alter the meaning of "densely approached" in a rather natural way, we may conclude, according to Theorem VI, that $f$ is densely approached everywhere except at the points of a set of measure zero. The new definition of dense approach is as follows: $f$ is said to be densely approached at $P$ if for every $\epsilon>0$ and for every non-vanishing partial neighborhood $N_{<}$of $P$, there is in $N_{<}$a point different from $P$ where $f$ differs from $f(P)$ by less than $\epsilon$.

Let now the relation $\Re_{r_{1} r_{2}}, r_{1}<r_{2}$, be such that $I \Re_{r_{1} r_{2}} P$ when and only when there is a point $(x, y, \zeta), r_{1} \leqq \zeta \leqq r_{2}$, inexhaustibly approached via $I$; that is to say, for every $\epsilon>0$ and for every circle $C$ of the $X Y$ plane with $P$ as center, there is an inexhaustible set of points in $C I$ at which $f \operatorname{differs}$ from $\zeta$ by less than $\epsilon$. Then it follows that $\Re_{r_{1} r_{2}}$ is closed. If $f$ is inexhaustibly approached at $P$ and a non-vanishing partial neighborhood $N_{<}$of $P$ exists via which $f$ is not inexhaustibly approached at $P$, then there exist two rational numbers $r_{1}$ and $r_{2}, r_{1}<f(P)<r_{2}$, such that $N \Re_{r_{1} r_{2}} P$ for every neighborhood $N$ of $P$ and $\overline{N_{<} \Re_{r_{1} r_{2}} P}$. Therefore according to Lemma II, $P$ belongs to a certain set $Z_{r_{1} r_{2}}$ of measure zero associated with the closed relation $\Re_{r_{1} r_{2}}$. The sum of the $Z_{r_{1} r_{2}}$ for all possible rational numbers $r_{1}<r_{2}$ is also of measure zero; we thus have

TheOREM VII. The set of points $P$ of the $X Y$ plane, at which f is inexhaustibly approached and for which a non-vanishing partial neighborhood exists via which $f$ is exhaustibly approached at $P$, constitute a set of measure zero.

It is, of course, not true that the points where $f$ is exhaustibly approached form a set of measure zero. Because of this fact the result for inexhaustible 
approach is not as pleasing as that for the case we shall consider next. We shall say that $f$ is neglectably approached at the point $P$, if a sphere $S$ exists with $P^{\prime}$ as center such that the projection upon the $X Y$ plane of the points of the surface that lie in $S$ constitutes a set of zero measure; $f$ is said to be neglectably approached at the point $P$ via $N_{<}$if a sphere $S$ exists with $P^{\prime}$ as center such that the projection upon the $X Y$ plane of $N_{<}^{\prime} S$ has in common with $N_{<}$a set of measure zero. We have the following

TheOREM VIII. Let $z=f(x, y)$ be any real, single-valued function defined in the entire $X Y$ plane. Then the points $P$ of the $X Y$ plane that possess a nonvanishing partial neighborhood via which $f$ is neglectably approached at $P$ constitute a set of zero measure.

The proof of this theorem, which is a generalization of Theorem VI, is analogous to that of the latter. A few indications will therefore suffice. We note first that the set of points where $f$ is neglectably approached is of measure zero; the proof is similar to that of the exhaustibility of the set of points at which $f$ is exhaustibly approached.* We then define $I \Re_{r_{1} r_{2}} P, P \equiv(x, y)$, as follows: there is a point $(x, y, \zeta), r_{1} \leqq \zeta \leqq r_{2}$, such that if $S$ is any sphere with $(x, y, \zeta)$ as center, and the surface points in $S$ are projected upon the $X Y$ plane, the portion falling in $I$ forms a set of positive exterior measure; i.e., $(x, y, \zeta)$ is not neglectably approached via $I$. It will be seen that $\Re_{r_{1} r_{2}}$ is closed. The rest of the argument is left to the reader.

Theorem VIII asserts that certain sets are of positive exterior measure. We shall go a little further by considering the question of exterior metric density. Let $P$ be any point and $M_{P e}$ the set of points $Q$ for which

$$
|f(Q)-f(P)|<\epsilon .
$$

We shall say that $f$ is quasi-continuous $\dagger$ at $P$ if for every $\epsilon$ the set $M_{P \epsilon}$ has 1 as exterior metric density at $P$. We have the following theorem, which generalizes Theorem VIII:

THEOREM IX. $f$ is quasi-continuous except at the points of a set of measure zero.

Proof. Let $S_{r_{1} r_{2}}, r_{1}<r_{2}$, be the set of points $(x, y)$ for which

$$
r_{1}<f(x, y)<r_{2},
$$

and $T_{r_{1} r_{2}}$ be the set of points where the exterior metric density of $S_{r_{1} r_{2}}$ is not 1; $T_{r_{1} r_{2}}$ is therefore of measure zero according to Theorem V. If $r_{1}$ and $r_{2}$ are rational, we have in all $\aleph_{0}$ sets $T_{r_{1} r_{2}}$, and thus the points in all the $T_{r_{1} r_{2}}$ constitute a set $Z$ of zero measure. If $P$ is a point at which $f$ is not quasi-continuous, then, according to definition, there exists a positive number $\epsilon$ such that

* See Section 1.

†Cf. Denjoy, Bulletin de la Société Mathématique de France, vol. 43 (1915), p. 165. 
$M_{P e}$ is not of exterior metric density 1 at $P$. Let $r_{1}$ and $r_{2}$ be two rational numbers such that

$$
f(P)-\epsilon<r_{1}<f(P)<r_{2}<f(P)+\epsilon .
$$

It follows that $P$ belongs to $T_{r_{1} r_{2}}$ and therefore to $Z$.

Suppose now that $N_{<}$is a partial neighborhood of $P$ such that if $C_{r}$ is a circle with $P$ as center and $r$ as radius then

$$
\liminf _{r \rightarrow 0} \frac{m\left(C_{r} N_{<}\right)}{m\left(C_{r}\right)}=k>0 ;
$$

the symbol $m$ denotes Lebesgue measure, which $N_{<}$, as an $I$-region, possesses. We shall then say that $N_{<}$is a "properly non-vanishing partial neighborhood" of $P$. Suppose further that a positive $\epsilon$ exists such that

$$
\liminf _{r \rightarrow 0} \frac{m_{e}\left(C_{r} N_{<} M_{P_{e}}\right)}{m\left(C_{r} N_{<}\right)}=l<1 .
$$

We shall then say that the exterior metric density of $M_{P e}$ is $\neq 1$ at $P$ via $N_{<}$. It follows then that the surface $z=f(x, y)$ is not quasi-continuous at $P$. For if $\delta$ is any positive number, there exists a circle $C_{r}$ with arbitrarily small radius and with $P$ as center such that

and

$$
m\left(C_{r} N_{<}\right)>(k-\delta) m\left(C_{r}\right),
$$

Therefore

$$
m_{e}\left(C_{r} N_{<} M_{P \varepsilon}\right)<(l+\delta) m\left(C_{r} N_{<}\right) .
$$

$$
\begin{aligned}
m_{e}\left(C_{r} M_{P e}\right)= & m_{e}\left(C_{r} N_{<} M_{P e}\right)+m_{e}\left(C_{r}\left\{C_{r}-N_{<}\right\} M_{P e}\right) \\
& <[(l+\delta)(k-\delta)+1-(k-\delta)] m\left(C_{r}\right) \\
= & {[1-(1-l-\delta)(k-\delta)] m\left(C_{r}\right) }
\end{aligned}
$$

for sufficiently small $\delta$; $C_{r}-N_{<}$means here the portion of $C_{r}$ not in $N_{<}$. Since $l<1, k>0$, and $\delta$ and $r$ may be made as small as we please, we conclude that the relative exterior measure of $M_{P e}$ is not 1 at $P$; that is, that $f$ is not quasi-continuous at $P$. The totality of such points $P$ is therefore of measure zero. We thus have

Theorem $\mathrm{X}$. If $P$ is a point not belonging to a certain set of measure zero, then for every positive $\epsilon$ the exterior metric density of $M_{P e}$ is unity at $P$ via any properly non-vanishing partial neighborhood of $P$.

Since an angular region at $P$, i.e., the portion of the $X Y$ plane bounded by two half lines radiating from $P$, is a properly non-vanishing partial neighborhood of $P$, it follows as a corollary of Theorem $\mathrm{X}$ that, if we neglect a set of measure zero, the exterior metric density of $M_{P \varepsilon}$ is, for every $\epsilon>0$, equal to unity at $P$ via every angular region with $P$ as vertex.

Trans. Am. Math. Soc. 9. 


\section{CONCLUding REMARKS}

As in the case of the descriptive properties, it may be seen that the metric theorems may be extended to many-valued functions. Theorem VIII, for example, when thus generalized, would read as follows: Let $z=f(x, y)$ be any real, single- or many-valued function defined in the entire $X Y$ plane. Then the points $(x, y)$ of the $X Y$ plane for which a surface point $(x, y, f(x, y))$ and a non-vanishing partial neighborhood $N_{<}$exist such that $(x, y, f(x, y))$ is neglectably approached via $N_{<}$constitute a set of zero measure.

It is evident that the metric properties hold for functions of a single variable and, in general, for functions of $n$ variables. Extension to $\aleph_{0}$-space and to function space would require a satisfactory definition of measure for such spaces; ${ }^{*}$ it is not our purpose to enter upon such questions here. Likewise we shall not attempt to define, by means of postulates, a general space for which our metric results are to hold.

It may be remarked that instead of projecting the surface points of $z=f(x, y)$ upon the $X Y$ plane we may project them upon the $X$-axis and thus obtain other properties. For example, let us define the relationship $\Re_{r_{1} r_{2} r_{3} r_{4}}$ (between $I$-regions and points of the $X$-axis) as follows: $I \Re_{r_{1} r_{2} r_{3} r_{4}} \xi$ if the surface points having $x$-coördinates in $I$ have a limit point in the rectangle $x=\xi, r_{1} \leqq y \leqq r_{2}, r_{3} \leqq z \leqq r_{4} . \Re_{r_{1} r_{2} r_{3} r_{4}}$ is evidently closed. If the $r$ 's are taken to be rational, the number of possible relations $\Re_{r_{1} r_{2} r_{3} r_{4}}$ is $\boldsymbol{\aleph}_{0}$, and we may, by the aid of Lemma II and an argument repeatedly employed in this paper, obtain the following result: Let $z=f(x, y)$ be any single- or many-valued function defined in the entire $X Y$ plane. Let $\xi$ be a point of the $X$-axis of the following character: a surface point $(\xi, \eta, \zeta)$ and a partial nonvanishing (linear) neighborhood $N_{<}$of $\xi$ exist such that $(\xi, \eta, \zeta)$ is not a limit point of surface points having $x$-coördinates in $N_{<}$. The totality of points $\xi$ is of measure zero.

Similar results may be obtained for other metric properties and also in the case of the descriptive properties. In the case of a function of $n$ variables, we may project upon an $(n-1)$-space, an $(n-2)$-space, etc.

* In this connection cf. Gâteaux, Bulle tin de la S o ciét é $\mathrm{M}$ a thé matique de France, vol. 47 (1919), p. 47.

UNIVERSITY OF ILLINOIS, Urbana, Ill. 